\title{
Hypoxic Tumor-Derived Exosomes Induce M2 Macrophage Polarization via AMPK/p38 To Promote Lung Cancer Progression
}

\section{Shiyu Zhou}

Third Affiliated Hospital of Guangzhou Medical College

\section{Yu Lan}

Third Affiliated Hospital of Guangzhou Medical College

\section{Yuqun Li}

Third Affiliated Hospital of Guangzhou Medical College

\section{Zhenxing Li}

Third Affiliated Hospital of Guangzhou Medical College

\section{Jinding Pu}

Third Affiliated Hospital of Guangzhou Medical College

\section{Liping Wei ( $\nabla$ weilp8@hotmail.com )}

Third Affiliated Hospital of Guangzhou Medical College

\section{Research Article}

Keywords: lung cancer, hypoxia, exosomes, macrophage, metastasis, PKM2/AMPK

Posted Date: June 1st, 2021

DOI: https://doi.org/10.21203/rs.3.rs-507408/v1

License: (1) This work is licensed under a Creative Commons Attribution 4.0 International License. Read Full License 


\section{Abstract \\ Background}

Hypoxia is a major regulator of tumor aggressiveness and metastasis in cancer progression. Exosomes (exos) play an important role in the communication between lung cancer and hypoxic microenvironment. However, the underlying mechanisms are largely undefined.

\section{Methods}

Exos isolated from A549 cells under hypoxia conditions. Transmission electron microscopy and nanoparticle tracking analysis were carried out to characterize exos. CCK-8 assay, flow cytometry, Western blot, wound healing and transwell assays were performed to assess the proliferation, apoptosis, migration, and invasion of A549 cells, respectively. The M2 polarization of macrophages was evaluated by RT-qPCR and Western blot analysis. In vivo nude mice model was established to determine the regulatory effect of hypoxia/exos on the progression of lung cancer.

\section{Results}

Hypoxic A549 cell-derived exos (hypoxia/exos) promoted the proliferation and migration, and inhibited the apoptosis in $\mathrm{A} 549$ cells. The expression of PKM2 was significantly upregulated in hypoxia/exos. Hypoxic exosomal PKM2 induced M2 polarization of macrophages by activating AMPK/p38 pathway. Co-culture with hypoxia/exos-treated macrophages enhanced the migration, invasion, and epithelialmesenchymal transition (EMT) in A549 cells. Moreover, treatment with hypoxia/exos facilitated the tumor growth and lung metastasis of A549 cells.

\section{Conclusions}

Our findings reveal that hypoxic exosomal PKM2 induces M2 macrophage polarization via AMPK/p38 pathway, and thus exerts a simulative effect on the growth and metastasis of lung carcinoma.

\section{Introduction}

Lung cancer is the most common malignancy worldwide and its mortality ranks first (1). Despite the advance in the current therapies, the 5-year survival of petients with lung cancer remains unsatisfactory (2). Therefore, identification of novel diagnostic/therapeutic targets is urgently needed.

Intertumoral hypoxia is a dynamic and heterogeneous feature of most solid tumors, which drives multiple cancer processes, such as cell proliferation, epithelial-mesenchymal transition (EMT), invasion, and metastasis (3). Hypoxia regulates the cellular communication and crosstalk between tumor cells and 
their microenvironment via diverse secretory factors, including exosomes (exos) (4). Exos are vesicles of endocytic origin ranging 30-150nm in diameter and carry packages of molecular information, including proteins, nucleic acids, lipids, and metabolites wrapped in their lipid bilayer (5). Accumulated evidence demonstrates that exos derived from hypoxic tumor cells contribute to cancer progression by promoting tumor cell proliferation, migration, and invasion and conferring chemo-resistance (6-8).

Macrophages are one of the most abundant cells with noteworthy plasticity in tumor microenvironment (9). Macrophages could be polarized to classically activated M1 and alternatively activated M2 macrophages, depending on the microenvironmental signals (10). M1 macrophages exert proinflammatory phenotype and tumor cytotoxicity, while M2 macrophages suppress the inflammation, contributing to angiogenesis and favoring tumor progression $(11,12)$. However, the association and mechanism of macrophages polarization involved in lung cancer progression remain to be elucidated.

In this study, we investigated the effect of hypoxic A549 cells-derived exos on the biological behavior of A549 cells and found that hypoxia/exos could promote the proliferation and migration of lung cancer cells both in vitro and in vivo. Moreover, we found that hypoxic exosomal PKM2 induced M2 polarization of macrophages through AMPK/p38 pathway, thus contributing to the promotion of tumor progression.

\section{Methods}

\section{Cell culture and hypoxia treatment}

A549 and RAW264.7 cells were obtained from the American Type Culture Collection (Manassas, VA, USA) and cultured in DMEM medium with $10 \%$ fetal calf serum (FBS) and $1 \%$ penicillin/streptomycin at $37^{\circ} \mathrm{C}$ in an atmosphere containing $5 \% \mathrm{CO}_{2}$. Hypoxia environment was established with a hypoxia cell incubator of $1 \% \mathrm{O}_{2}$.

\section{Exos isolation and identification}

A549 cells were cultured under normoxic $\left(21 \% \mathrm{CO}_{2}\right)$ or hypoxic $\left(1 \% \mathrm{O}_{2}\right)$ conditions for 3 days. The medium was replaced with $10 \%$ exos-depleted FBS at $80-90 \%$ confluence. Then the medium was collected and centrifuged at $300 \mathrm{~g}$ for $10 \mathrm{~min}, 3000 \mathrm{~g}$ for $20 \mathrm{~min}, 10,000 \mathrm{~g}$ for $30 \mathrm{~min}$, and ultra-centrifuged at $100,000 \times \mathrm{g}$ for $2 \mathrm{~h}$ at $4{ }^{\circ} \mathrm{C}$ to precipitate exos pellets. After the pellets were resuspended in PBS and stained with the phosphotungstic acid for $10 \mathrm{~min}$, transmission electron microscopy experiment was performed for exos identification (13).

\section{Nanoparticle tracking analysis}

The nanoparticle relative concentrations were analyzed using the Nanosight (Malvern, Worcestershire, UK) and NTA analytical software (version 2.3, Nanosight) (14).

\section{Western blot analysis}


Cells were harvested and ultra-centrifuged at $100,000 \times \mathrm{g}$ for $30 \mathrm{~min}$ at $4^{\circ} \mathrm{C}$ to collect proteins. The concentration of proteins was determined with the BCA protein assay kit (Thermo Fisher; Waltham, MA, USA). $20 \mu \mathrm{g}$ protein was loaded on an SDS-PAGE, separated by electrophoresis, and transferred to PVDF membrane. The membranes were blocked with $5 \%$ skimmed milk for $1 \mathrm{~h}$ and incubated with specific antibodies overnight at $4^{\circ} \mathrm{C}$ subsequently. Monoclonal antibodies (Abcam; Cambridge, UK) used in this study were as follows: CD9 (1:1000), CD81 (1:1000), TSG101 (1:1000), HSP70 (1:1000), ALIX (1:1000), Cleaved-caspase3 (1:1000), bcl-2 (1:1000),bax (1:1000), PKM2 (1:1000), p-P38 (1:1000), p38 (1:1000), pAMPK (1:1000), AMPK (1:1000), CD206 (1:1000), Arginase-1 (1:1000), E-cadherin (1:1000), N-cadherin (1:1000), Vimentin (1:1000) and GAPDH (1:10,000). The membranes were incubated with HRPconjugated secondary antibodies before being visualized using a Chemiluminescent HRP Substrate Kit (Millipore; Billerica, MA, USA).

\section{CCK-8 assay}

A549 cells $\left(1 \times 10^{4} /\right.$ well) were seeded into 96 -well plates and treated with normoxia/exos or hypoxia/exos for $24 \mathrm{~h}, 48 \mathrm{~h}$, and $72 \mathrm{~h}$. Then $10 \mu \mathrm{l} \mathrm{CCK}-8$ solution (Dojindo; Kumamoto, Japan) was added to each well and incubated at $37^{\circ} \mathrm{C}$ for $2 \mathrm{~h}$. The optical density (OD) value at $450 \mathrm{~nm}$ was measured with an Epoch microplate spectrophotometer (BioTek; Winooski, VT, USA) and cell viability was calculated.

\section{Wound-healing assay}

A549 cells were seeded into 6-well plates. Then cells were scratched with a 200- $\mu$ l sterile pipette tip at the confluence of $90 \%$. The wounded monolayers were washed with PBS to remove cell debris and A549 cells were cultured with fresh serum-free medium with normoxia/exos or hypoxia/exos. The distance between the two edges of the wound was calculated at $0 \mathrm{~h}, 24 \mathrm{~h}, 48 \mathrm{~h}$ to evaluate the migration capacity.

\section{Cell apoptosis analysis}

Cell apoptosis was examined with the Annexin V-FITC apoptosis detection kit (BD Biosciences; New Jersey, USA). After treatment with normoxia/exos or hypoxia/exos, A549 cells were collected and resuspended in $1 \times$ binding buffer, followed by staining with Annexin V-FITC and PI in the dark at room temperature. Then the percentage of apoptotic cells was immediately analyzed by a flow cytometer (BD, Biosciences; New Jersey, USA).

\section{Exo uptake assay}

After being labeled with the PKH67 Green Fluorescent Cell Linker Kit (Sigma-Aldrich; St. Louis, MO, USA), exos were added to RAW264.7 cells. Then, RAW264.7 cells were fixed with 4\% paraformaldehyde and stained with DAPI prior to its observation by a fluorescence microscopy.

\section{Real-Time quantitative PCR (RT-qPCR)}


Total RNA was isolated with the TRIzol reagent (Invitrogen; Carlsbad, CA, USA) according to the manufacturer's instructions. Reverse transcription reaction was performed by using reverse transcriptase kit (Transgen Biotech; Beijing, China). Subsequently, PCR was performed with a standard SYBR Green PCR kit (Transgen Biotech). GAPDH expression was regarded as the normalization control.

\section{Migration and invasion assays}

Migration and invasion assays were performed using transwell chambers (24 wells) (Corning; NY, USA) coated without or with Matrigel (BD Biosciences), respectively. $5 \times 10^{4}$ A549 cells were re-suspended in serum-free medium and placed in the top chamber. The lower chamber was filled with $1 \times 10^{4}$ macrophages induced by hypoxic/exos and normoxia/exos in $600 \mu$ medium containing $10 \%$ FBS. After incubation for $24 \mathrm{~h}$, the migrating or invading cells were fixed with methyl alcohol and subsequently stained with $0.1 \%$ crystal violet. The number of migrating or invading cells was counted using an Inverted microscope.

\section{Hematoxylin-eosin (HE) staining}

Suspicious lung metastasis tissues were assessed by HE staining. The tissues were placed in $10 \%$ formalin overnight, and then dehydrated and embedded in paraffin. Then the tissue samples were sliced to $4 \mu \mathrm{m}$ thick sections, fixed on a glass slide, dried and stained. The sections were successively immersed in xylene, ethanol with gradient concentrations, and hematoxylin. After being mounted with resin, the sections were dried naturally, and observed and photographed with a light microscope.

\section{Animal experiments}

All animal experiments were approved by the Guangzhou Medical University. Nude mice (male, 4-6 weeks of age, 18-20 g) were purchased from Guangdong Animal Experiment Center. Luciferase-labelled A549 cells were subcutaneously injected with into the oxter of nude mice. After the tumor grew to $50-100 \mathrm{~mm}^{3}$, mice were randomly divided into 3 groups: control, normoxia/exos, and hypoxia/exos $(n=5)$. Exos derived from A549 cells under normoxia or hypoxia conditions were injected into mice via the tail vein for the treatment. In vivo fluorescence imaging was performed to visualize the tumor growth. Tumors size was measured using a caliper weekly. Four weeks after injection, mice were sacrificed under general anesthesia. For metastasis, A549 cells were injected into nude mice via the tail vein. $24 \mathrm{~h}$ after injection, mice were treated with exos via tail vein injection. Suspicious lung metastasis tissues were analyzed by HE staining.

\section{Statistical analysis}

All in vitro results were derived from at least three independent experiments. Statistical analyses, histograms drawing, and scatter plots were performed with SPSS 22.0 software (SPSS Inc. USA) and GraphPad Prism 6.0 software (GraphPad Software, Inc.) The significance of differences between two groups was determined using Student's $t$-test. Comparations among more than two groups were 
performed with one-way analysis of variance (ANOVA). $P<0.05$ was considered to indicate a statistically significant difference.

\section{Results}

\section{Characterization of exos from A549 cells under normoxia and hypoxia}

A549 cells were placed under normoxia and hypoxia for $48 \mathrm{~h}$ and exos were isolated from the conditioned media. The exo morphology was identified by transmission electron microscope assay. The exos were presented as cup-shaped double-layer membrane structure ranging from 30 to150 nm in diameter (Fig. 1A). Nanoparticle tracking analysis showed that the nanoparticle relative concentration of exos derived from hypoxic A549 cells was significantly higher than that from normoxic condition (Fig. 1B). Hypoxia exos exhibited the elevated expression of exo specific markers (CD9, CD81, TSG-101, HSP70 and ALIX), as characterized by Western blot (Fig 1C).

\section{Hypoxia/exos promote the proliferation and migration, and inhibit apoptosis in A549 cells}

Next, we explored the effect of hypoxia/exos on A549 cells. As shown in Fig. 2A, A549 cells cultured with hypoxia/exos exhibited higher proliferation capacity than those cultured with normoxia/exos. Wound healing assay demonstrated that hypoxia/exos enhanced the migration ability of A549 cells (Fig. 2B). Moreover, flow cytometry analysis showed that administration of hypoxia/exos inhibited the apoptosis of A549 cells, compared with normoxia/exos (Fig. 2C). In line with these observations, the expression of cleaved-caspase3 and Bax was lower in the hypoxia/exo group than the normoxia/exo group (Fig. 2D), while the level of $\mathrm{Bcl} 2$ was increased in the hypoxia/exo group compared with the normoxia/exo group. These data indicated that hypoxia/exos could facilitated the proliferation and migration, and antagonized the apoptosis in A549 cells.

\section{Hypoxia/exos induced the polarization of M2 macrophages}

M2 macrophages are beneficial for cancer progression. We then investigated whether hypoxia/exos had an effect on macrophage polarization. The uptake assay showed that PKH67-labeled exos could be internalized by RAW264.7 cells (Fig. 3A). M1 macrophages are characterized by the expression of iNOS and IL-1 $\beta$, while M2 macrophages are characterized by the expression of CD206, CD163, TGF- $\beta$, IL-10, and Arginase-1. The results of RT-qPCR showed that the mRNA expression of CD206, CD163, TGF- $\beta$, IL-10, Arginase-1 was significantly higher in macrophages incubated with hypoxia/exos than those incubated with normoxia/exos (Fig. 3B). Consistently, the protein levels of M2 markers (CD206 and Arginase-1) were increased following the treatment with hypoxia/exos (Fig. 3C). Together, these results indicated that hypoxia/exos could induce macrophage towards to the M2 phenotype.

Glucose metabolism is important change in cancer cells under a hypoxic environment. PKM2 has been reported to play a critical role in glycolysis $(15,16)$. Interestingly, we found that the expression of PKM2 in hypoxia/exo was significantly increased compared with normoxia/exo (Fig. 3D). Western bolt results 
manifested higher levels of p-AMPK, and p-P38 in macrophages following stimulation with hypoxia/exos (Fig. 3E). Thus, these results implied that hypoxia/exos induces M2 polarization is associated with exosomal PKM2-mediated AMPK/p38 activation.

\section{Hypoxia/exos-induced M2 macrophages favor the migration, invasion, and EMT in A549 cells}

To determine the effects of M2 macrophages on the migration and invasion ability of A549 cells, we performed transwell assay. The results revealed that A549 cells co-incubated with M2 macrophages treated with hypoxia/exos exhibited superior migration and invasion abilities compared with the control cells (Fig. 5A and 5B). It is known that re-activation of the EMT program promotes cancer progression and enhances the metastatic phenotype (17). Western blot analysis presented that the expression of Ecadherin, a major epithelial marker, was decreased, while the expression levels of mesenchymal cell markers $\mathrm{N}$-cadherin and vimentin were enhanced in A549 co-cultured with hypoxia/exo-treated macrophages (Fig. 5C). All together, these results confirmed a more superior role of M2 macrophages induced by hypoxia/exos in promoting migration, invasion, and EMT in A549 cells.

\section{Hypoxia/exos facilitate tumor growth and metastasis of A549 cells in vivo}

Given the important role of hypoxa/exos in the proliferation and migration of A549 cells in vitro, we further verified the function of hypoxia/exos using the xenograft model via injection with luciferaselabeled A549 cells. Higher fluorescence intensity and increased tumor volume and tumor weight were observed in the hypoxia/exos group compared with the normoxia/exos group (Fig. 6A-C), indicating that hypoxia/exos exacerbated progressive growth in vivo. In addition, more lung metastatic nodules were observed in hypoxia/exos-treated mice compared with the control mice (Fig. 6D and 6E). Collectively, these data suggest that hypoxia/exos have a promoting effect on lung cancer progression.

\section{Discussion}

Hypoxia is a common phenomenon in the tumor microenvironment and is associated with tumor progression and drug resistance (3). Accumulating studies have been indicated that hypoxia triggers exos release from cancer cells, thus facilitating tumor progression (18). Herein, we isolated the exos from A549 cells under hypoxia conditions and investigated their function and mechanism in lung cancer progression. Our results showed that hypoxia/exos have an oncogenic activity in lung cancer, which is associated with exosomal PKM2-mediated induction of M2 macrophages via AMPK/p38 signaling.

Exos secreted by multiple cells have been reported to involve in tumorous biological function and exosomal concentration/cargo can be altered by hypoxia (4). Previous studies have been demonstrated that the acceleration of tumor progression was closely associated with the exos released from hypoxia tumor cells. For example, it has been reported that ovarian cancer cells exposed to hypoxia significantly increase their exos release and treatment with hypoxia-induced exos lead to a more aggressive and chemoresistant ovarian cancer phenotype (6). In this work, we also found that culture under hypoxic conditions favored the release of exos. Hypoxia/exos favored the proliferation and migration of A549 
cells in vitro. Also, treatment with hypoxia/exos significantly promoted the tumor growth and metastasis of A549 cells in vivo, indicating an oncogenic activity of hypoxia/exos in lung cancer.

It is well acknowledged that macrophages act as scavengers, modulating the immune response and maintaining tissue homeostasis (19). Macrophages can be reprogramed by metabolites, cytokines, and other signaling mediators, which makes them the key participants in tumor microenvironment and associated with enhanced tumor progression (20). It has been reported that M2 macrophage polarization could be regulated by exos. Bardi et al. have showed that exos released from melanoma cells induced a mixed M1 and M2 pro-tumor macrophages (21). Exos from adipose-derived stem cells polarize M2 macrophages and thus attenuate adipose inflammation and obesity (22). In the present study, the results showed that hypoxia/exos induced M2 macrophage polarization and therefore facilitated the migration and invasion of A549 cells.

Glucose metabolism change is a hallmark of cancer under hypoxia conditions. PKM2 is a key enzyme involved in glycolysis regulation and has an important regulatory role in cancer progression/resistance under hypoxia conditions (23-26). It has been reported that exosomal PKM2 secreted by hypoxic cisplatin-resistance cells transmitted cisplatin-resistance to sensitive non-small cell lung cancer (NSCLC) cells both in vitro and in vivo (27). In present study, we found that hypoxia/exos derived from A549 cells could significantly elevated PKM2 expression. AMPK has been reported to play an essential role M2 macrophage polarization $(28,29)$. Consistently, our data showed treatment with hypoxia/exos significantly increased the phosphorylation of AMPK and its downstream p38 in macrophages.

Collectively, our results implied that hypoxic exosmal PKM2 induces M2 macrophages, which was related to activation of AMPK/p38 pathway.

\section{Conclusions}

Our findings show that hypoxia/exos induced M2 macrophage polarization via AMPK/p38 pathway and thus promotes lung tumor growth and metastasis. This study manifests hypoxia/exos as the possible targets for fighting against cancer progression and metastasis.

\section{Abbreviations}

EMT epithelial-mesenchymal transition

NSCLC non-small cell lung cancer

Exos Exosomes

\section{Declarations}

\section{Ethics statement}


All animal experiments were approved by the Ethics Committee of Guangzhou Medical University 『GZXK2020-06)

\section{Consent for publication}

Not applicable

\section{Competing interests}

The authors declare that they have no competing interests

\section{Availability of data and materials}

The data that support the findings of this study are available from the corresponding author [email: weilp8@hotmail.com] upon reasonable request

\section{Funding}

This work was financially supported by the Guangzhou Health Science and Technology Project (NO.20201A011093)

\section{Authors' contributions}

(I) Conception and design: LY and ZSY; (II) Administrative support: LYQ; (III) Provision of study materials or patients:LZX ; (IV) Collection and assembly of data: LY and ZSY; (V) Data analysis and interpretation: LY and ZSY; (VI) Manuscript writing: All authors; (VII) Final approval of manuscript: All authors.

\section{Acknowledgments}

Not applicable

\section{References}

1. Bray, F., Ferlay, J., Soerjomataram, I., Siegel, R. L., Torre, L. A., and Jemal, A. (2018) Global cancer statistics 2018: GLOBOCAN estimates of incidence and mortality worldwide for 36 cancers in 185 countries. CA: a cancer journal for clinicians $68,394-424$

2. Socinski, M. A., Obasaju, C., Gandara, D., Hirsch, F. R., Bonomi, P., Bunn, P. A., Jr., Kim, E. S., Langer, C. J., Natale, R. B., Novello, S., Paz-Ares, L., Perol, M., Reck, M., Ramalingam, S. S., Reynolds, C. H., Spigel, D. R., Wakelee, H., and Thatcher, N. (2018) Current and Emergent Therapy Options for Advanced Squamous Cell Lung Cancer. Journal of thoracic oncology : official publication of the International Association for the Study of Lung Cancer 13, 165-183

3. Schito, L., and Semenza, G. L. (2016) Hypoxia-Inducible Factors: Master Regulators of Cancer Progression. Trends in cancer 2, 758-770 
4. Kumar, A., and Deep, G. (2020) Hypoxia in tumor microenvironment regulates exosome biogenesis: Molecular mechanisms and translational opportunities. Cancer letters 479, 23-30

5. Mashouri, L., Yousefi, H., Aref, A. R., Ahadi, A. M., Molaei, F., and Alahari, S. K. (2019) Exosomes: composition, biogenesis, and mechanisms in cancer metastasis and drug resistance. Molecular cancer 18,75

6. Dorayappan, K. D. P., Wanner, R., Wallbillich, J. J., Saini, U., Zingarelli, R., Suarez, A. A., Cohn, D. E., and Selvendiran, K. (2018) Hypoxia-induced exosomes contribute to a more aggressive and chemoresistant ovarian cancer phenotype: a novel mechanism linking STAT3/Rab proteins. Oncogene 37, 3806-3821

7. Xue, M., Chen, W., Xiang, A., Wang, R., Chen, H., Pan, J., Pang, H., An, H., Wang, X., Hou, H., and Li, X. (2017) Hypoxic exosomes facilitate bladder tumor growth and development through transferring long non-coding RNA-UCA1. Molecular cancer 16, 143

8. Zhang, X., Sai, B., Wang, F., Wang, L., Wang, Y., Zheng, L., Li, G., Tang, J., and Xiang, J. (2019) Hypoxic BMSC-derived exosomal miRNAs promote metastasis of lung cancer cells via STAT3-induced EMT. Molecular cancer 18, 40

9. Najafi, M., Hashemi Goradel, N., Farhood, B., Salehi, E., Nashtaei, M. S., Khanlarkhani, N., Khezri, Z., Majidpoor, J., Abouzaripour, M., Habibi, M., Kashani, I. R., and Mortezaee, K. (2019) Macrophage polarity in cancer: A review. Journal of cellular biochemistry 120, 2756-2765

10. Sica, A., and Mantovani, A. (2012) Macrophage plasticity and polarization: in vivo veritas. The Journal of clinical investigation 122, 787-795

11. Xiang, W., Shi, R., Kang, X., Zhang, X., Chen, P., Zhang, L., Hou, A., Wang, R., Zhao, Y., Zhao, K., Liu, Y., Ma, Y., Luo, H., Shang, S., Zhang, J., He, F., Yu, S., Gan, L., Shi, C., Li, Y., Yang, W., Liang, H., and Miao, H. (2018) Monoacylglycerol lipase regulates cannabinoid receptor 2-dependent macrophage activation and cancer progression. Nature communications 9,2574

12. Gordon, S., and Martinez, F. O. (2010) Alternative activation of macrophages: mechanism and functions. Immunity 32, 593-604

13. Wu, X., Showiheen, S. A. A., Sun, A. R., Crawford, R., Xiao, Y., Mao, X., and Prasadam, I. (2019) Exosomes Extraction and Identification. Methods in molecular biology (Clifton, N.J.) 2054, 81-91

14. Mao, Y., Wang, Y., Dong, L., Zhang, Y., Zhang, Y., Wang, C., Zhang, Q., Yang, S., Cao, L., Zhang, X., Li, X., and $\mathrm{Fu}, \mathrm{Z}$. (2019) Hypoxic exosomes facilitate angiogenesis and metastasis in esophageal squamous cell carcinoma through altering the phenotype and transcriptome of endothelial cells. Journal of experimental \& clinical cancer research : CR 38, 389

15. Yang, Y. C., Chien, M. H., Liu, H. Y., Chang, Y. C., Chen, C. K., Lee, W. J., Kuo, T. C., Hsiao, M., Hua, K. T., and Cheng, T. Y. (2018) Nuclear translocation of PKM2/AMPK complex sustains cancer stem cell populations under glucose restriction stress. Cancer letters 421, 28-40

16. Liu, M., Zhang, Z., Wang, H., Chen, X., and Jin, C. (2019) Activation of AMPK by metformin promotes renal cancer cell proliferation under glucose deprivation through its interaction with PKM2. International journal of biological sciences $15,617-627$ 
17. Chaffer, C. L., San Juan, B. P., Lim, E., and Weinberg, R. A. (2016) EMT, cell plasticity and metastasis. Cancer metastasis reviews 35, 645-654

18. King, H. W., Michael, M. Z., and Gleadle, J. M. (2012) Hypoxic enhancement of exosome release by breast cancer cells. BMC cancer 12, 421

19. Mehla, K., and Singh, P. K. (2019) Metabolic Regulation of Macrophage Polarization in Cancer. Trends in cancer 5, 822-834

20. Nielsen, S. R., and Schmid, M. C. (2017) Macrophages as Key Drivers of Cancer Progression and Metastasis. Mediators of inflammation 2017, 9624760

21. Bardi, G. T., Smith, M. A., and Hood, J. L. (2018) Melanoma exosomes promote mixed M1 and M2 macrophage polarization. Cytokine 105, 63-72

22. Zhao, H., Shang, Q., Pan, Z., Bai, Y., Li, Z., Zhang, H., Zhang, Q., Guo, C., Zhang, L., and Wang, Q. (2018) Exosomes From Adipose-Derived Stem Cells Attenuate Adipose Inflammation and Obesity Through Polarizing M2 Macrophages and Beiging in White Adipose Tissue. Diabetes 67, 235-247

23. Luo, W., Hu, H., Chang, R., Zhong, J., Knabel, M., O'Meally, R., Cole, R. N., Pandey, A., and Semenza, G. L. (2011) Pyruvate kinase M2 is a PHD3-stimulated coactivator for hypoxia-inducible factor 1. Cell $145,732-744$

24. Schwartz, L., Supuran, C. T., and Alfarouk, K. O. (2017) The Warburg Effect and the Hallmarks of Cancer. Anti-cancer agents in medicinal chemistry 17, 164-170

25. Wong, N., Ojo, D., Yan, J., and Tang, D. (2015) PKM2 contributes to cancer metabolism. Cancer letters 356, 184-191

26. Li, Y. H., Li, X. F., Liu, J. T., Wang, H., Fan, L. L., Li, J., and Sun, G. P. (2018) PKM2, a potential target for regulating cancer. Gene $668,48-53$

27. Wang, D., Zhao, C., Xu, F., Zhang, A., Jin, M., Zhang, K., Liu, L., Hua, Q., Zhao, J., Liu, J., Yang, H., and Huang, G. (2021) Cisplatin-resistant NSCLC cells induced by hypoxia transmit resistance to sensitive cells through exosomal PKM2. Theranostics 11, 2860-2875

28. Ye, W., Wang, J., Lin, D., and Ding, Z. (2020) The immunomodulatory role of irisin on osteogenesis via AMPK-mediated macrophage polarization. International journal of biological macromolecules 146, 25-35

29. Xu, F., Cui, W. Q., Wei, Y., Cui, J., Qiu, J., Hu, L. L., Gong, W. Y., Dong, J. C., and Liu, B. J. (2018) Astragaloside IV inhibits lung cancer progression and metastasis by modulating macrophage polarization through AMPK signaling. Journal of experimental \& clinical cancer research : CR 37, 207

\section{Figures}


A

Hypoxia/exos

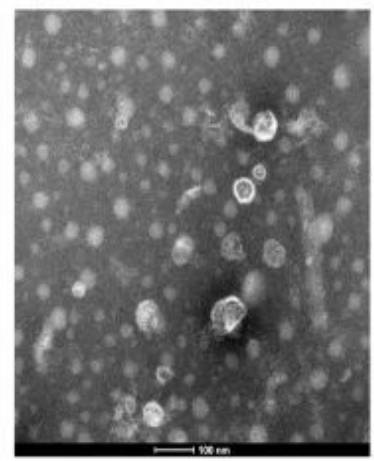

Normoxia/exos

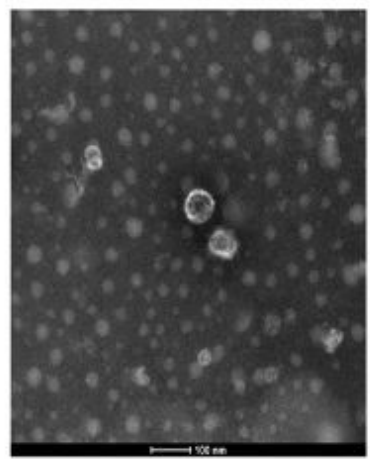

B

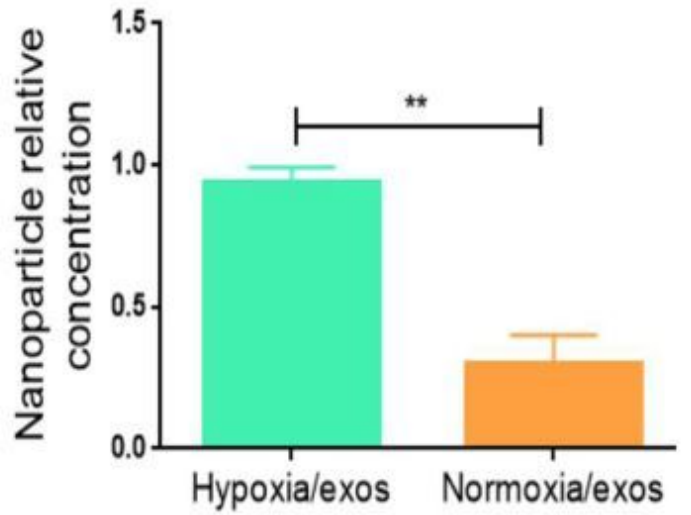

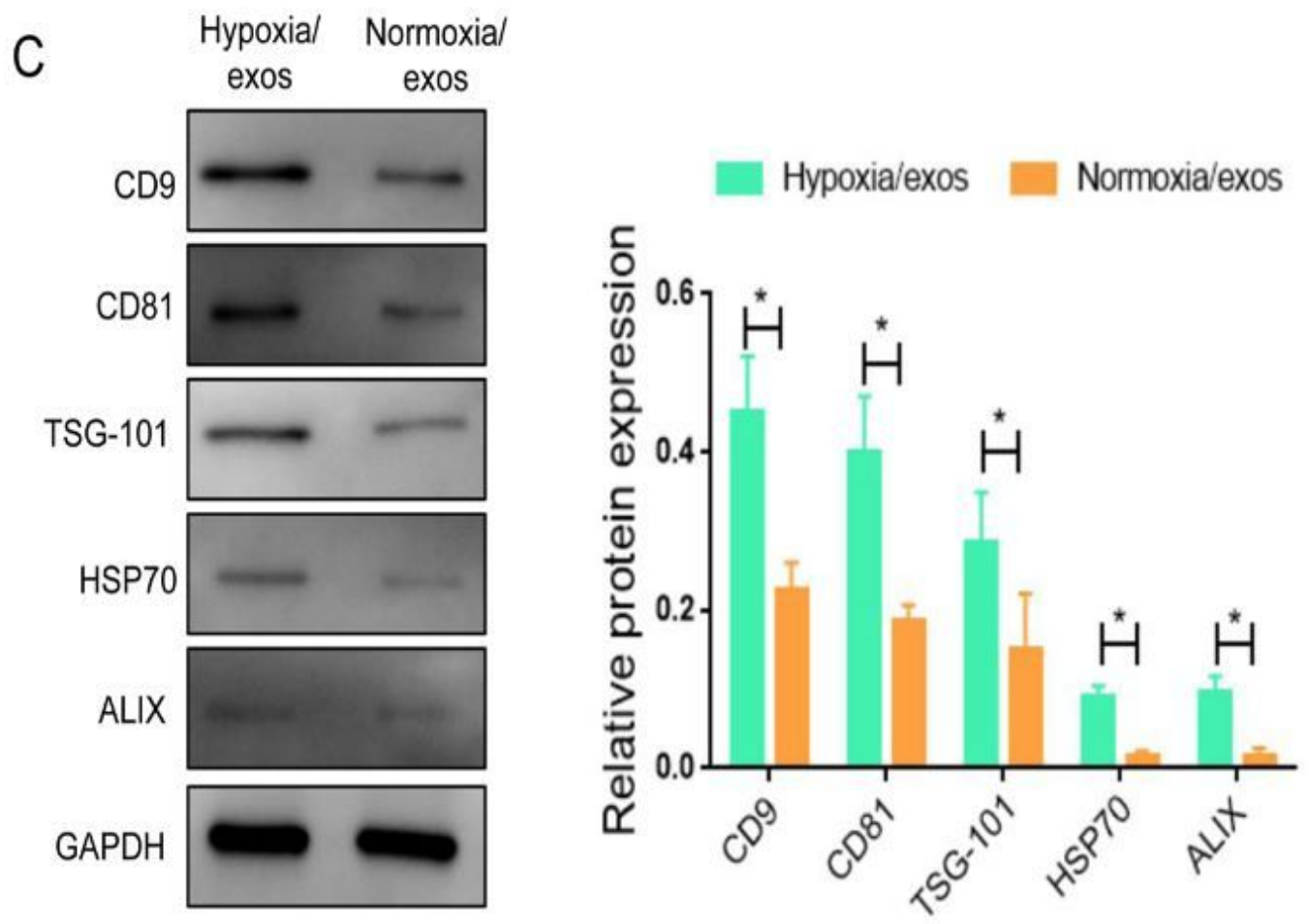

\section{Figure 1}

Characterization of exos from A549 cells under normoxia and hypoxia conditions. A. Exos were identified by transmission electron microscope. B. Identical concentrations of A549 cells were seeded under normoxia and hypoxia conditions and the nanoparticle relative concentration was determined. C. Western blot analysis of marker proteins of exos (CD9, CD81, TSG101, HSP70, ALIX). ${ }^{\star} P<0.05$; ${ }^{\star \star} P<0.01$. 
A

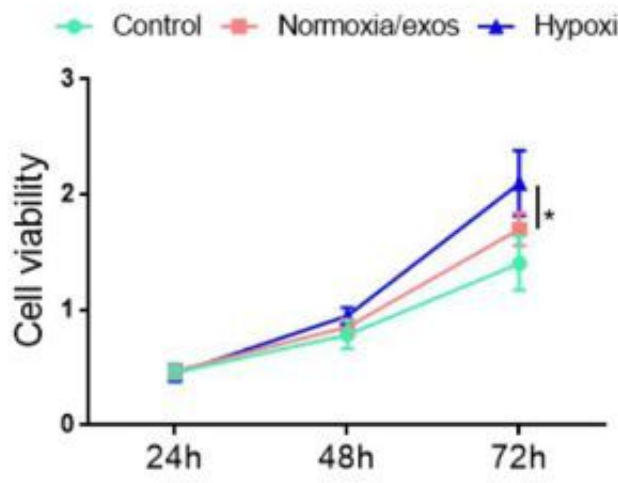

B
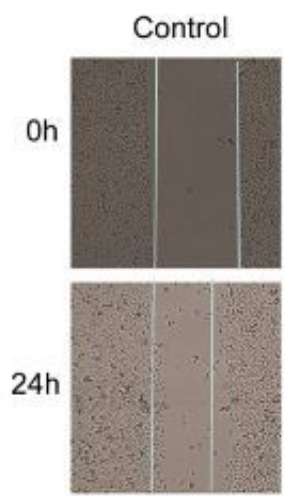

$48 \mathrm{~h}$

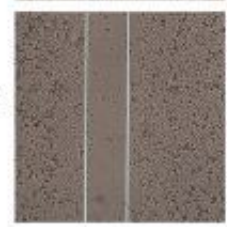

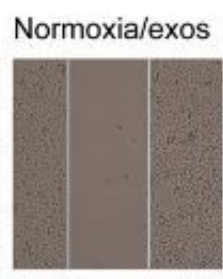
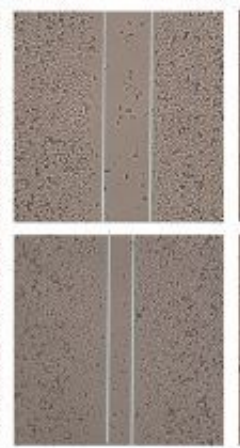

Hypoxia/exos
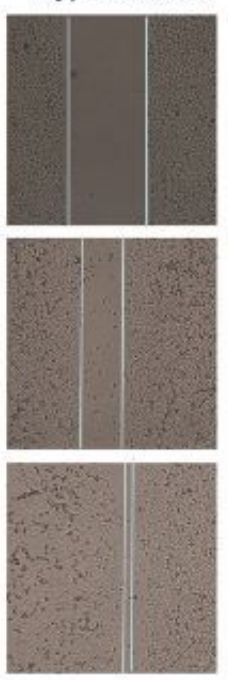

C
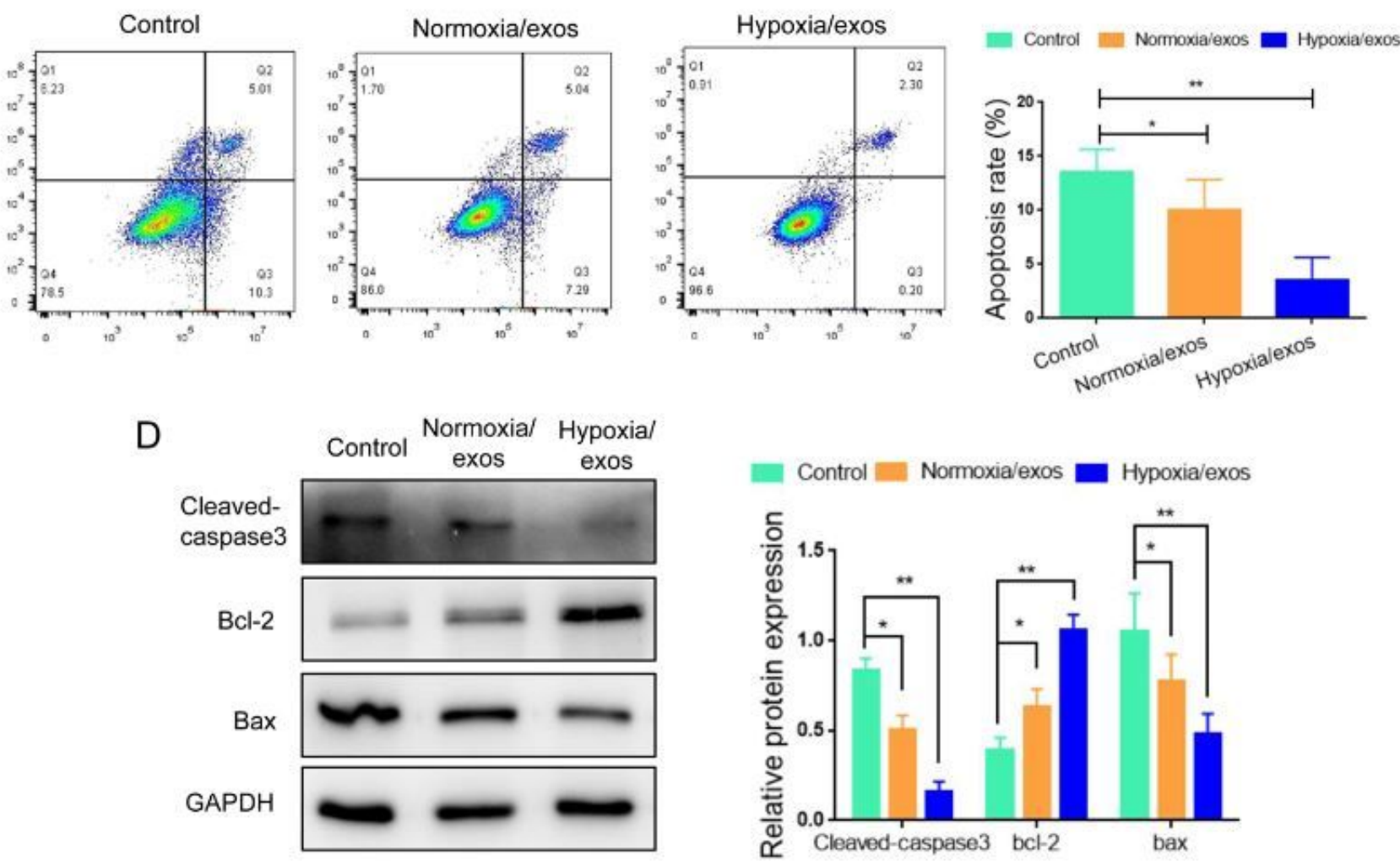

\section{Figure 2}

Hypoxia/exos promotes the proliferation and inhibited apoptosis in A549 cells. A. The proliferation of A549 cells after treatment with hypoxia/exos and normoxia/exos was determined by CCK-8 assay. B. The migration of A549 cells was assessed by wound healing assay. C. The apoptosis of A549 cells was detected by flow cytometry analysis. D. The protein levels of cleaved-caspase $3, \mathrm{Bcl}-2$ and Bax were determined by Western blot. ${ }^{*} \mathrm{P}<0.05 ;{ }^{* *} \mathrm{P}<0.01$. 
A

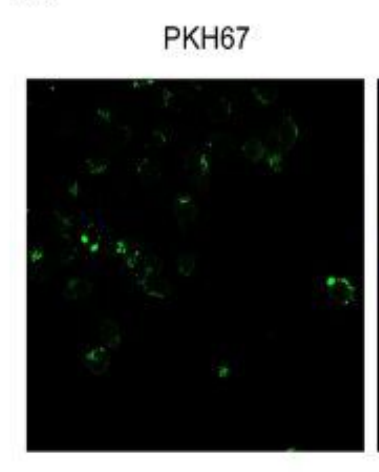

B

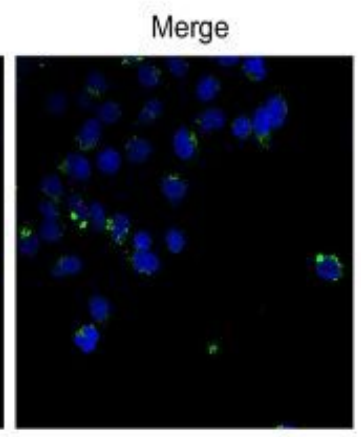

Control $[$ Normoxia/exos Hypoxia/exos

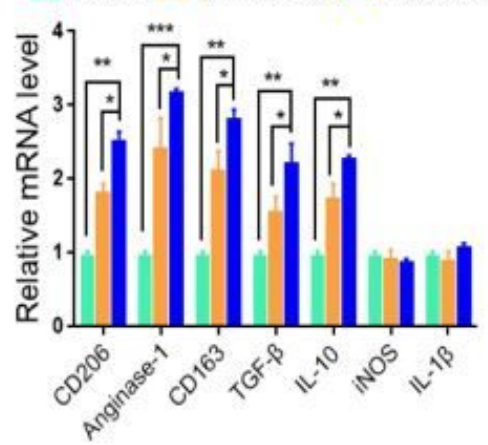

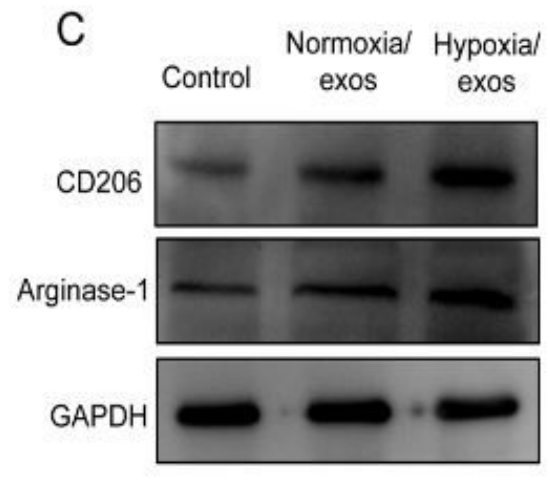

D
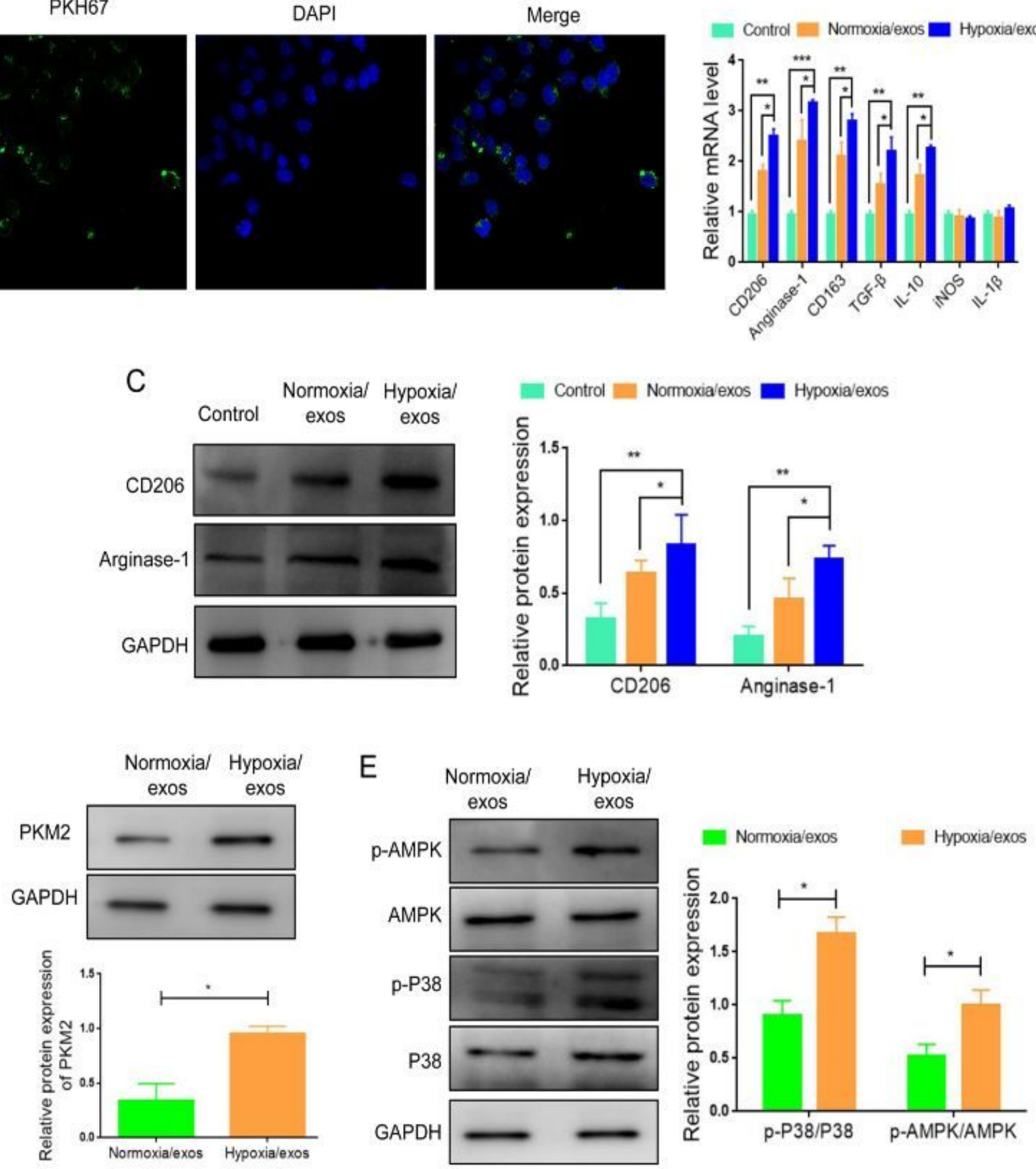

\section{Figure 3}

Hypoxia/exos induce the polarization of M2 macrophages. A. Immunofluorescence staining displayed the internalization of hypoxia/exos (green) by RAW264.7 cells. B. RT-qPCR analysis for M2 markers (CD206, CD163, TGF- $\beta$, IL-10, Arginase-1) and M1 markers (iNOS, IL-1 $\beta$ ). C. Western blot analysis of the protein expression of M2 markers (CD206 and Arginase-1). D. Western blot analysis of PKM2 expression in hypoxia/exos or normoxia/exo. E. Western blot analysis of the protein and phosphorylation levels of AMPK and p38 in macrophages exposed to hypoxia/exos or normoxia/exos. ${ }^{*} \mathrm{P}<0.05$; ${ }^{\star \star} \mathrm{P}<0.01$; $* \star \star \mathrm{P}<$ 0.001 . 


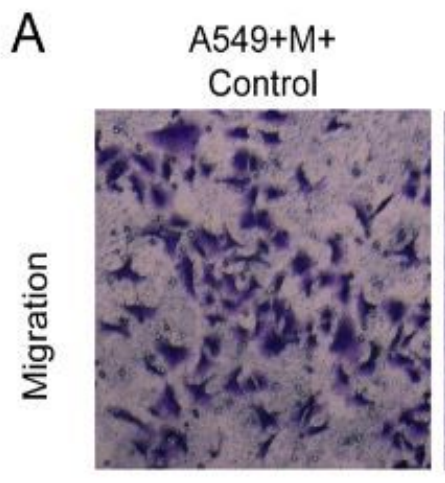

A549+M+ Normoxia/exos

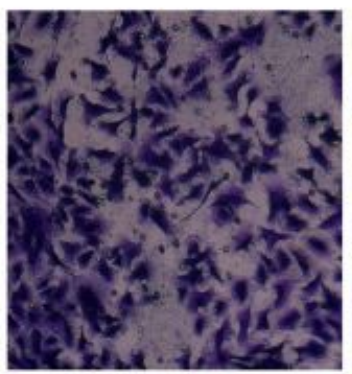

A549+M+ Hypoxia/exos
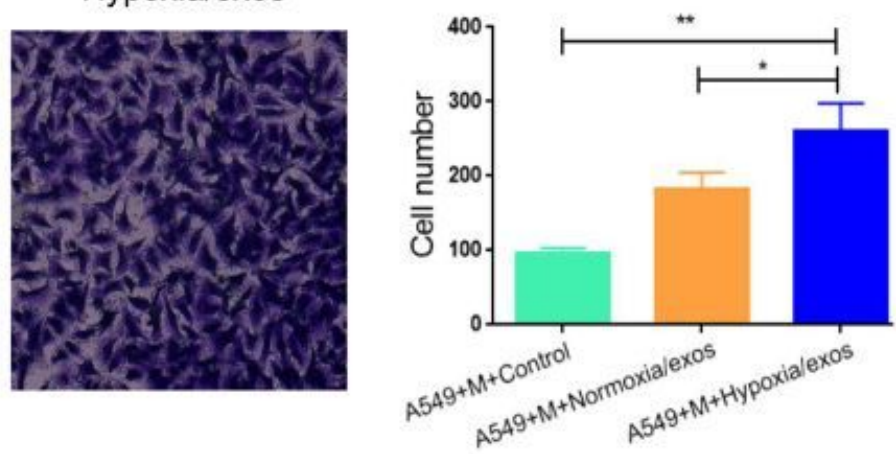

B
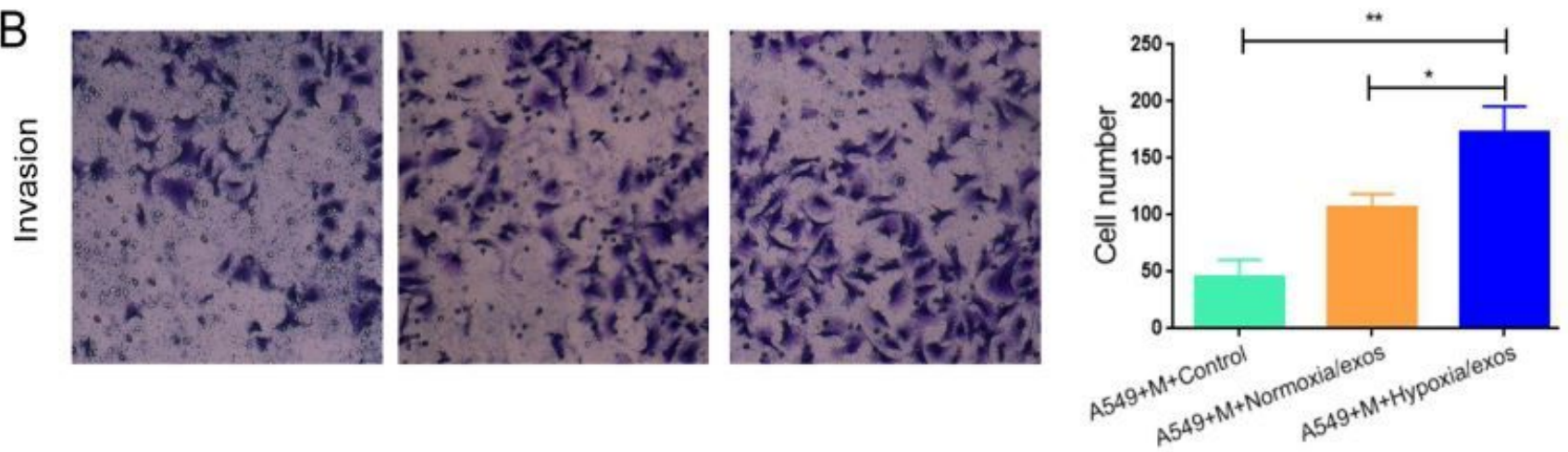

\section{C \\ A549+M+ \\ A549+M+ \\ A549+M+ \\ Contro Normoxia/exos \\ Hypoxia/exos}
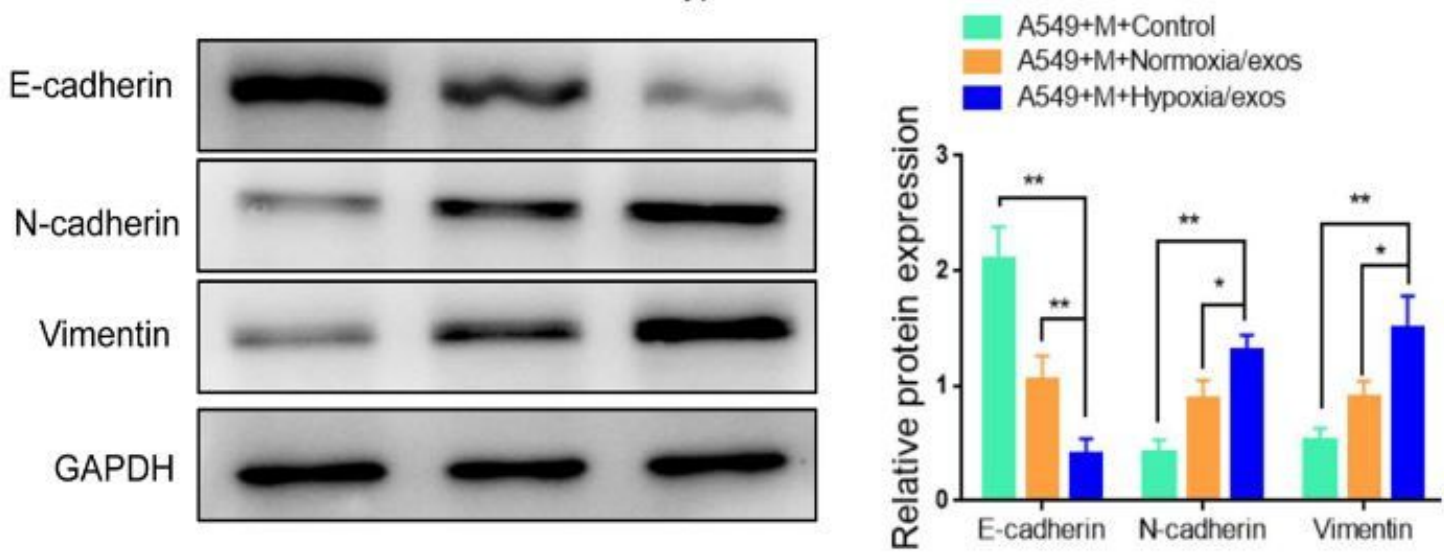

\section{Figure 4}

M2 macrophages polarized by hypoxia/exos promote the migration, invasion, and EMT in A549 cells. A. Transwell migration assay in A549 cells co-cultured with hypoxia/exos (or normoxia/exo)-treated macrophages. B. Transwell invasion assay in A549 cells co-cultured with hypoxia/exos (or normoxia/exo)-treated macrophages. C. Western blot analysis of EMT related proteins (E-cadherin, Ncadherin, Vimentin). M, RAW264.7 macrophages; * $\mathrm{P}<0.05 ; * \star \mathrm{P}<0.01$. 
A

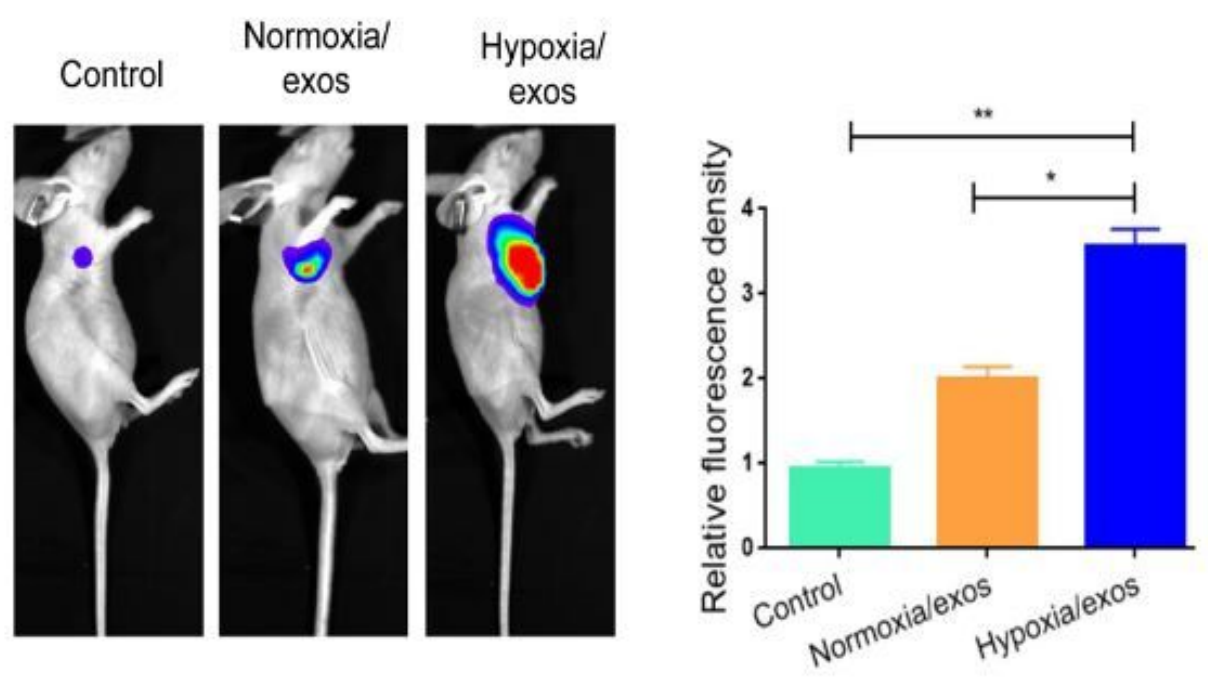

B

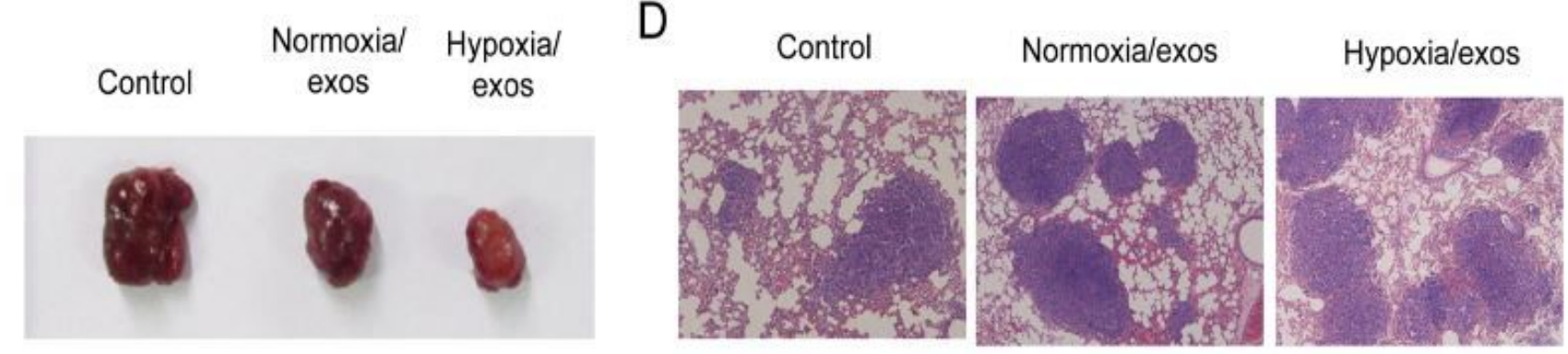

C
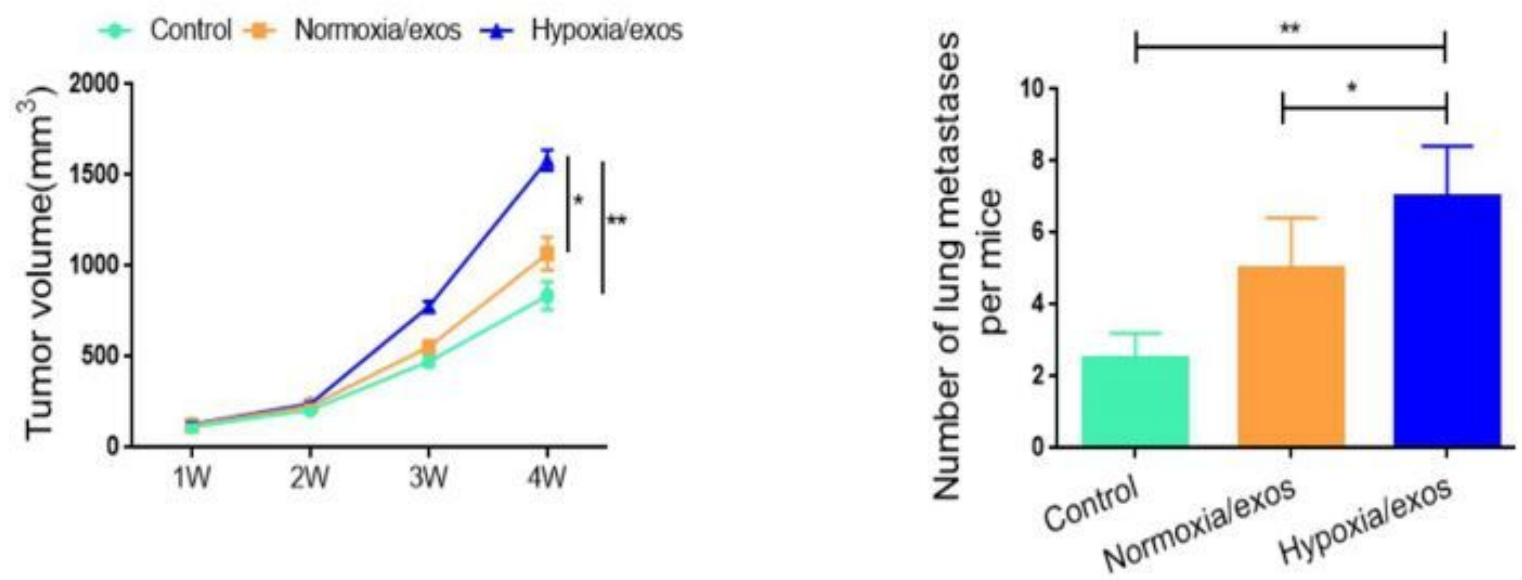

\section{Figure 5}

Hypoxia/exos facilitate the tumor growth and metastasis of A549 cells in vivo. A. In vivo fluorescence imaging for detecting the tumor growth of A549 cells. B. The growth curve of tumor growth in mice after treatment with hypoxia/exos (or normoxia/exo) for 4 weeks. C. The tumor weight of tumors isolated from nude mice treated with hypoxia/exos (or normoxia/exo). D. HE staining of lung metastasis nodules. $\mathrm{E}$. The number of metastatic nodules in $\mathrm{D}$. ${ }^{*} \mathrm{P}<0.05$; $*$ $\mathrm{P}<0.01$. 